\title{
Fault Tolerant Control Based on PID-type Fuzzy Logic Con- troller for Switched Discrete-time Systems: An Electronic Throt- tle Valve Application
}

\author{
Wafa Gritli ${ }^{*}$ Hajer Gharsallaoui, Mohamed Benrejeb
}

Université de Tunis El Manar, LA.R.A Automatique, Ecole Nationale d'Ingénieurs de Tunis, BP 37, Le Belvédère, 1002 Tunis, Tunisie.

\begin{tabular}{l} 
A R T I C L E I N F O \\
\hline Article history: \\
Received: 09 November, 2017 \\
Accepted: 01 December, 2017 \\
Online: 14 December, 2017 \\
\hline Keywords: \\
Electronic throttle valve \\
Switched systems \\
Fault tolerant control \\
PID-type FLC \\
Unknown input observer \\
Lyapunov theory \\
LMIs
\end{tabular}

\section{Introduction}

Actuator faults may cause undesired system behaviour and sometimes lead to instability, hence it is necessary to develop Fault Tolerant Control (FTC) methods against actuator faults of uncertain nonlinear systems. In the past decades some FTC design methods have been proposed for several classes of nonlinear systems with actuator faults [1-3]. It consists in computing control laws by taking into account the faults affecting the system in order to maintain acceptable performances and to preserve stability of the system in the faulty situations [4]. From the point of view of FTC strategies, the literature considers two main groups of techniques: passive and active ones. In passive FTC, the faults are treated as uncertainties. Therefore, the control is designed to be robust only to the specified faults [4]. Active FTC techniques consist in adapting the control law using the information given by the Fault Detection and Isolation (FDI) block [5]. The informations issued from the FDI block are used by the FTC module to reconfigure the control law in order to compensate the fault and to ensure an acceptable system performances.

The study of this problem was extended to switched systems in [6-8]. In [7], a switched discrete-time system with state delay has been considered. The design method is based on the construction of a filter and a fault estimation approach. In [8], an adaptive fuzzy tracking control method for a class of switched nonlinear systems with arbitrary switchings and with actuator faults has been proposed. The proposed control scheme guarantee the stability of the whole switched control system based on the common Lyapunov function stability theory and attenuate the effect of the actuator faults on the control performance by designing a new fuzzy controller to accommodate uncertain actuator faults. In [9], an observer has been built to detect the fault when it occurs. The problem of FTC for switchied linear systems is addressed by using a nominal control law designed in the absence of any fault, associated with fault detection, localization and reconfiguration techniques to maintain the stability of the system under an arbitrary switching signal in the presence of sensor faults. A state trajectory tracking has been proposed in [4] for actuator faults and observer bank based on controllers with switching mechanism for sensor faults has been also presented in [10]. A nonlinear observer based controller, adopting the so-

${ }^{*}$ Corresponding Author: Wafa Gritli, Email: wafa.gritli@enit.rnu.tn 
called parallel distributed compensation structure, has been designed to choose an adequate state estimate to compensate the effects of the faults on the system in [11]. Switched systems are dynamical systems consisting of a collection of continuous-time subsystems. Switched systems have attracted more attention due to their significance in the modelling of many engineering applications, such as chemical processes, robot manipulators and power systems. Stability analysis and synthesis of switched systems have been made using Lyapunov function to ensure stability of the switched systems such in [12].

In this paper, in order to acheive the FTC for a switched discrete-time systems, a set of PID-type Fuzzy Logic Controller (FLC) is implemented to minimize the error between the desired flat trajectory and the estimated state and to maintain the stability of the system in the presence of actuator faults. The estimated state is provided from an Unknown Input Observer (UIO). Based on residual analysis, a switching strategy using stateflow is then designed. The global stability for the switched systems is studied by Lyapunov theory and expressed as a Linear Matrix Inequalities (LMIs).

The paper is organized as follows. In Section 2, the FTC problem statement is formulated. In Section 3, the fault detection method using UIO is introduced. In Section 4, the PID-type FLC is described. Section 5 deals with the flatness property. The proposed approach is applied to an ETV in Section 6. Finally, the conclusion is drawn in Section 7.

\section{FTC problem statement}

Consider the discrete time switched system, which can be formulated such that

$$
\begin{gathered}
x(k+1)=A_{j} x(k)+B_{j} u(k)+E_{j} d(k)+B_{j} f_{a}(k) \\
y(k)=C_{j} x(k)
\end{gathered}
$$

$x(k) \in \mathbb{R}^{n}$ is the state vector, $u(k) \in \mathbb{R}^{p}$ the control input, $y(k) \in \mathbb{R}^{o}$ the measured output, $d(k) \in \mathbb{R}^{p}$ the unknown disturbance input and $f_{a}(k) \in \mathbb{R}^{p}$ the actuator faults. $A_{j} \in \mathbb{R}^{n \times n}, B_{j} \in \mathbb{R}^{n \times p}, C_{j} \in \mathbb{R}^{o \times n}$ and $E_{j} \in \mathbb{R}^{n \times p}$ are the known constant matrices for $j \in \psi=\{1,2, \ldots, m\}$ and $m$ the number of models, $m>1$.

In this paper, a FTC structure based on PID-type FLC, given by Figure 1, is proposed to maintain the trajectory tracking and to preserve stability of ETV in the presence of both input disturbance $d(k)$ and actuator faults $f_{a}(k)$.

According to this structure, the FTC approach needs to detect actuator faults firstly and then to design the $j^{\text {th }}$ control law, denoted $u_{j}(k)$ given by $(2)$, in order to minimize the error between the desired flat trajectory, generated using the flatness property and the estimated state, provided from the $j^{\text {th }}$ UIO.

$$
u_{j}(k)=-K_{c, j} \hat{x}_{j}(k)+y_{j}^{d}(k)
$$

$K_{c, j}$ is the $j^{\text {th }}$ gain control law.

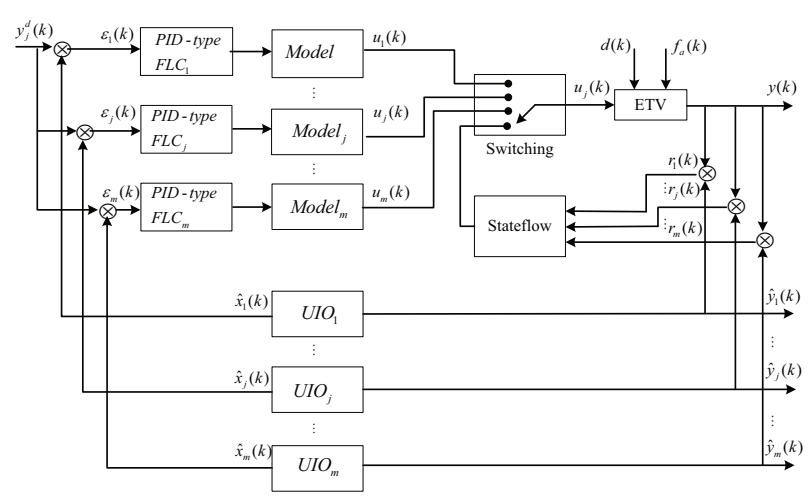

Figure 1: Proposed structure of FTC approach

In this case, LMI-based UIOs for the switched system (1) are designed using Lyapunov function for arbitary switching signal. Then, residuals $r_{j}(k)$ are determined by the UIOs and employed to achieve actuator faults detection.

\section{Unknown input observers design and stability analysis}

In this section, let us consider $f_{a}(k)=0$, then the model (1) becomes

$$
\begin{gathered}
x(k+1)=A_{j} x(k)+B_{j} u(k)+E_{j} d(k) \\
y(k)=C_{j} x(k)
\end{gathered}
$$

$j \in \psi$ denotes the $j^{\text {th }}$ model. The structure of full rank UIOs can be formulated by

$$
\begin{gathered}
z(k+1)=F_{j}(k)+T_{j} B_{j} u(k)+K_{j} y(k) \\
\hat{x}(k)=z(k)+H_{j} y(k)
\end{gathered}
$$

where $\hat{x}(k)$ is the estimated state vector $x(k), z(k)$ is the state vector of full rank UIOs. $F_{j}, T_{j}, K_{j}$ and $H_{j}$ are unknown matrices which need to be designed.

Lemma: [13] Equation (4) is UIO of the switched system (3), if and only if the following conditions are satisfied

$$
\begin{aligned}
& \text { - } \operatorname{rank}\left(C_{j} E_{j}\right)=\operatorname{rank}\left(E_{j}\right) \\
& \text { - }\left(C_{j} A_{j 1}\right) \text { is observable }
\end{aligned}
$$

with

$$
A_{j 1}=A_{j}-E_{j}\left[\left(C_{j} E_{j}\right)^{T} C_{j} E_{j}\right]^{-1}\left(C_{j} E_{j}\right)^{T} C_{j} A_{j}
$$

According to the above, the formulation of UIOs is constructed for each subsystem. In the next part, the multiple Lyapunov function will be used to realize the design of parameters for UIOs of switched system.

Theorem: [14] In the condition of arbitrary switching signal, for the system (3), if

$$
\begin{gathered}
\left(H_{j} C_{j}-I\right) E_{j}=0 \\
T_{j}=\left(I-H_{j} C_{j}\right)
\end{gathered}
$$




$$
\begin{gathered}
F_{j}=\left(A_{j}-H_{j} C_{j} A_{j}-K_{j 1} C_{j}\right) \\
K_{j 2}=F_{j} H_{j}
\end{gathered}
$$

hold, and there exist symmetric matrices $P_{j}>0, \forall j \in$ $\psi$ such that

$$
\left[\begin{array}{cc}
-P_{j} & P_{j} F_{j} \\
F_{j}^{T} P_{j} & -P_{j}
\end{array}\right] \leqslant 0, \forall j \in \psi
$$

then the parameters of UIOs can be designed.

Proof: Define the state estimation error as $e(k)=$ $x(k)-\hat{x}(k)$, the dynamic equation can be derived as

$$
\begin{aligned}
& e(k+1)=\left(A_{j}-H_{j} C_{j} A_{j}-K_{j 1} C_{j}\right) e(k) \\
& -\left[F_{j}-\left(A_{j}-H_{j} C_{j} A_{j}-K_{j 1} C_{j}\right)\right] z(k) \\
& -\left[K_{j 2}-\left(A_{j}-H_{j} C_{j} A_{j}-K_{j 1} C_{j}\right) H_{j}\right] y(k) \\
& -\left[T_{j}-\left(I-H_{j} C_{j}\right)\right] B_{j} u(k)-\left(H_{j} C_{j}-I\right) E_{j} d(k)
\end{aligned}
$$

with

$$
K_{j}=K_{j 1}+K_{j 2}
$$

In order to make the error decoupled from known control input $u(k)$, measured output $y(k)$ and unknown input $d(k)$, we should let

$$
\begin{gathered}
\left(H_{j} C_{j}-I\right) E_{j}=0 \\
T_{j}-\left(I-H_{j} C_{j}\right)=0 \\
F_{j}-\left(A_{j}-H_{j} C_{j} A_{j}-K_{j 1} C_{j}\right)=0 \\
K_{j 2}-\left(A_{j}-H_{j} C_{j} A_{j}-K_{j 1} C_{j}\right) H_{j}=0
\end{gathered}
$$

It can be concluded that

$$
\begin{gathered}
H_{j}=E_{j}\left[\left(C_{j} E_{j}\right)^{T} C_{j} E_{j}\right]^{-1}\left(C_{j} E_{j}\right)^{T} \\
A_{j 1}=A_{j}-E_{j}\left[\left(C_{j} E_{j}\right)^{T} C_{j} E_{j}\right]^{-1}\left(C_{j} E_{j}\right)^{T} C_{j} A_{j} \\
F_{j}=A_{j}-H_{j} C_{j} A_{j}-K_{j 1} C_{j}=A_{j 1}-K_{j 1} C_{j}
\end{gathered}
$$

and the error dynamics is given by

$$
e(k+1)=F_{j} e(k)
$$

Equation (4) is UIOs of the switched system (3) if the estimation error tends asymptotically to zero despite the presence of an unknown input $d(k) \neq 0$.

Consider the following Lyapunov function

$$
V_{j}(e(k))=e(k)^{T} P_{j} e(k)
$$

For $P_{j}=P_{j}^{T}>0$, then, $V_{j}(e(k))>0$ holds, the $\Delta V$ given by $(16)$ is negative

$$
\begin{gathered}
V_{i}(e(k+1))-V_{j}(e(k)) \\
=e(k)^{T} F_{j}^{T} P_{i} F_{j} e(k)-e(k)^{T} P_{j} e(k) \\
=e(k)^{T}\left(F_{j}^{T} P_{i} F_{j}-P_{j}\right) e(k) ;(i \in \psi, j \in \psi, i \neq j)
\end{gathered}
$$

if the following inequalities are satisfied

$$
F_{j}^{T} P_{i} F_{j}-P_{j} \leqslant 0
$$

Thus, the error system (14) is stable asymptotically. According to Schur complement lemma, the inequalities 17) can be rewritten as

$$
\left[\begin{array}{cc}
-P_{i} & P_{i} F_{j} \\
F_{j}^{T} P_{i} & -P_{j}
\end{array}\right] \leqslant 0
$$

By substituting $F_{j}=A_{j 1}-K_{j 1} C_{j}$, the above matrix inequalities become

$$
\left[\begin{array}{cc}
-P_{i} & P_{i}\left(A_{j 1}-K_{j 1} C_{j}\right) \\
\left(A_{j 1}-K_{j 1} C_{j}\right)^{T} P_{i} & -P_{j}
\end{array}\right]<0
$$

and

$$
\left[\begin{array}{cc}
-P_{i} & \left(P_{i} A_{j 1}-W_{i j} C_{j}\right) \\
\left(P_{i} A_{j 1}-W_{i j} C_{j}\right)^{T} & -P_{j}
\end{array}\right]<0
$$

for $W_{i j}=P_{i} K_{j 1}$.

Since $K_{j 1}=P_{j}^{-1} W_{i j}$, we can obtain the value of $K_{j 1}$ then $F_{j}, K_{j 2}$ and $K_{j}=K_{j 1}+K_{j 2}$ from $P_{j}$ and $W_{i j}$ solutions of LMIs.

The aim of fault detection is to generate a residual signal $r_{j}(k)$, given by (21), which is sensitive to $f_{a}(k)$ in the presence of actuator faults which is the purpose of [15].

$$
r_{j}(k)=y(k)-C_{j} \hat{x}_{j}(k)
$$

\section{PID-type fuzzy logic controller de- sign}

In this study, the FTC approach is based on PID-type FLC. To adjust the input and the output scaling factors of this controller, Genetic Algorithm (GA) optimization technique has been proposed in order to improve the performance of the controller.

\subsection{PID-type fuzzy logic controller descrip- tion}

We consider a PID-type FLC structure as shown in Figure 2, [16], where $K_{e} \in \mathbb{R}^{+}$and $K_{d} \in \mathbb{R}^{+}$are the input scaling factors, $\alpha \in \mathbb{R}^{+}$and $\beta \in \mathbb{R}^{+}$the output scaling factors.

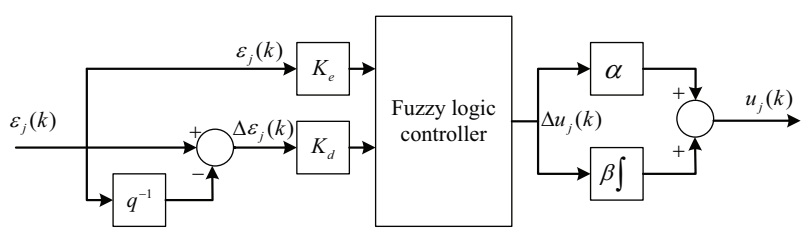

Figure 2: The PID-type FLC

The FLC inputs variables, the error $\varepsilon_{j}(k)$ between the desired flat trajectory $y_{j}^{d}(k)$ and the estimated state $\hat{x}(k)$, and error variation $\Delta \varepsilon_{j}(k)$ are given by the equations 22 and (23) where $T_{e}$ is the sampling period.

$$
\begin{gathered}
\varepsilon_{j}(k)=-K_{c, j} \hat{x}(k)+y_{j}^{d}(k) \\
\Delta \varepsilon_{j}(k)=\frac{\varepsilon_{j}(k)-\varepsilon_{j}(k-1)}{T_{e}}
\end{gathered}
$$

The output variable $\Delta u_{j}(k)$ of a such controller is the variation of the control law signal $u_{j}(k)$ which can be defined as 24 .

$$
\Delta u_{j}(k)=\frac{u_{j}(k)-u_{j}(k-1)}{T_{e}}
$$


The output of the PID-type FLC is given by [25), [17]

$$
\begin{aligned}
& u_{j}(k)=\alpha \Delta u_{j}(k)+\beta \int \Delta u_{j}(k) \mathrm{d} t \\
& =\alpha\left(A+Q K_{e} u_{j}(k)+D K_{d} \Delta u_{j}(k)\right) \\
& +\beta \int\left(A+Q K_{e} u_{j}(k)+D K_{d} \Delta u_{j}(k)\right) \mathrm{d} k \\
& =\alpha A+\beta A k+\left(\alpha K_{e} Q+\beta K_{d} D\right) u_{j}(k) \\
& +\beta K_{e} Q \int u_{j}(k) \mathrm{d} k+\alpha K_{d} D \Delta u_{j}(k)
\end{aligned}
$$

where the terms $Q$ and $D$ are given by (26) and (27), [10].

$$
\begin{aligned}
& Q=\frac{\Delta u_{(i+1) j}-\Delta u_{i j}}{\varepsilon_{i+1}-\varepsilon_{i}} \\
& D=\frac{\Delta u_{i(j+1)}-\Delta u_{i j}}{\Delta \varepsilon_{j+1}-\Delta \varepsilon_{j}}
\end{aligned}
$$

The fuzzy controllers with product-sum inference method, centroid defuzzification method and triangular uniformly distributed membership functions for the inputs and a crisp output proposed in [18], are used, in our study.

The linguistic levels, assigned to the variables $\varepsilon_{j}(k)$, $\Delta \varepsilon_{j}(k)$ and $\Delta u_{j}(k)$, are given in Table 1 as follows: $N L$ : Negative Large; $N$ : Negative; $Z R$ : Zero; $P$ : Positive; $P L$ : Positive Large.

\begin{tabular}{|c|c|c|c|}
\hline$\varepsilon_{j}(k) / \Delta \varepsilon_{j}(k)$ & $N$ & $Z R$ & $P$ \\
\hline$N$ & $N L$ & $N$ & $Z R$ \\
\hline$Z R$ & $N$ & $Z R$ & $P$ \\
\hline$P$ & $Z R$ & $P$ & $P L$ \\
\hline
\end{tabular}

Table 1: Fuzzy rules-base

\subsection{Optimization of scaling factors using ge- netic algorithm}

To adjust the input and the output scaling factors $\left(K_{e}\right.$, $\left.K_{d}\right)$ and $(\alpha, \beta)$, GA is used in order to obtain their optimal values.

At first, an initial chromosome population is randomly generated. The chromosomes are candidate solutions to the problem. Then, the fitness values of all chromosomes are evaluated by calculating an objective function. So, based on the fitness of each individual, a group of the best chromosomes is selected through the selection process. The genetic operators, crossover and mutation, are applied to this 'surviving' population in order to improve the next generation solutions. Crossover is a recombination operator that combines subparts of two parent chromosomes to produce offspring. This operator extracts common features from different chromosomes in order to achieve even better solutions. Mutation is an operator that introduces variations into the chromosome. The modifications can consist of changing one or more values of a chromosome. Through the mutation operator the search space is explored by looking for better points. The process continues until the population converges to the stop criterion.

The most crucial step in applying GA is to choose the objective function that is used to evaluate the fitness of each chromosome. In this paper, the method of tuning PID-type FLC parameters using GA is based on minimizing the Integral of the Squared Error (ISE) used in $[18]$.

\section{$5 \quad$ Flatness and trajectory planning}

The flatness approach is used in a discrete-time framework for system (1). Let the studied dynamic linear discrete system described by (28)

$$
D_{j}(q) y_{j}(k)=N_{j}(q) v_{j}(k)
$$

$q$ is the forward operator, $v_{j}(k)$ and $y_{j}(k)$ are, respectively, the input and the output signals and $D_{j}(q)$ and $N_{j}(q)$ the polynomials defined by

$$
\begin{array}{r}
D_{j}(q)=q^{n}+a_{j, n-1} q^{n-1}+\ldots+a_{j, 1} q+a_{j, 0} \\
N_{j}(q)=b_{j, n-1} q^{n-1}+\ldots+b_{j, 1} q+b_{j, 0}
\end{array}
$$

where the parameters $a_{j, l}$ and $b_{j, l}$ are constants, $l=$ $\{0,1, \ldots, n-1\}$.

The system is considered as linear and controllable, consequently it is flat, [16].

The flat output $z_{j}(k)$, on which depend the output $y_{j}(k)$ and the control $v_{j}(k)$, can be seen as being the partial state of a linear system, [19]

$$
\begin{aligned}
& v_{j}(k)=D_{j}(q) z_{j}(k) \\
& y_{j}(k)=N_{j}(q) z_{j}(k)
\end{aligned}
$$

The open loop control law can be determined by the following relations, [18]

$$
\begin{gathered}
v_{j}^{d}(k)=f\left(z_{j}^{d}(k), \ldots, z_{j}^{d^{(r+1)}}(k)\right) \\
y_{j}^{d}(k)=g\left(z_{j}^{d}(k), \ldots, z_{j}^{d^{(r)}}(k)\right)
\end{gathered}
$$

$f$ and $g$ are vectorial functions and $z_{j}^{d}(k)$ is the desired trajectory that must be differentiable at the $(r+1)$ order.

In order to plan the desired flat trajectory $z_{j}^{d}(k)$, the polynomial interpolation technique is used.

Let consider the state vector: $Z_{j}^{d}(k)=$ $\left(z_{j}^{d}(k) z_{j}^{d^{(1)}}(k) \ldots z_{j}^{d^{(r+1)}}(k)\right)^{T}$ containing the desired flat output and its successive derivatives, [18].

The expression of $Z_{j}^{d}(k)$ can be given as following where $k_{0}$ and $k_{f}$ are two moments known in advance.

$$
Z_{j}^{d}(k)=M_{j, 1}\left(k-k_{0}\right) c_{j, 1}\left(k_{0}\right)+M_{j, 2}\left(k-k_{0}\right) c_{j, 2}\left(k_{0}, k_{f}\right)
$$

such that

$$
M_{j, 1}=\left(\begin{array}{cccc}
1 & k & \cdots & \frac{k^{n-1}}{(n-1) !} \\
0 & 1 & \cdots & \frac{k^{(n-2)}}{(n-2) !} \\
\vdots & \ddots & \ddots & \vdots \\
0 & \cdots & 0 & 1
\end{array}\right)
$$




$$
\begin{gathered}
M_{j, 2}=\left(\begin{array}{cccc}
\frac{k^{n}}{n !} & \frac{k^{n+1}}{(n+1) !} & \cdots & \frac{k^{2 n-1}}{(2 n-1) !} \\
\frac{k^{n-1}}{(n-1) !} & \frac{k^{n}}{n !} & \cdots & \frac{k^{(n-2)}}{(n-2) !} \\
\vdots & \ddots & \ddots & \vdots \\
k & \cdots & \frac{k^{n-1}}{(n-1) !} & \frac{k^{n}}{n !}
\end{array}\right) \quad(37) \\
c_{j, 1}=Z_{j}^{d}\left(k_{0}\right) \\
c_{j, 2}=M_{j, 2}^{-1}\left(k_{f}-k_{0}\right)\left(Z_{j^{d}}\left(k_{f}\right)-M_{j, 1}\left(k_{f}-k_{0}\right) Z_{j}^{d}\left(k_{0}\right)\right)
\end{gathered}
$$

After planning a desired flat trajectory in discrete-time framework, the real output $y_{j}(k)$, to be controlled, follows the desired trajectory $y_{j}^{d}(k)$ such that 40, , [18].

$$
y_{j}^{d}(k)=N_{j}(q) z_{j}^{d}(k)
$$

\section{Application of an electronic throt- tle valve}

\subsection{Electronic throttle valve modeling}

The proposed approach is applied here for the case of the electronic throttle valve, Figure 3. [15].

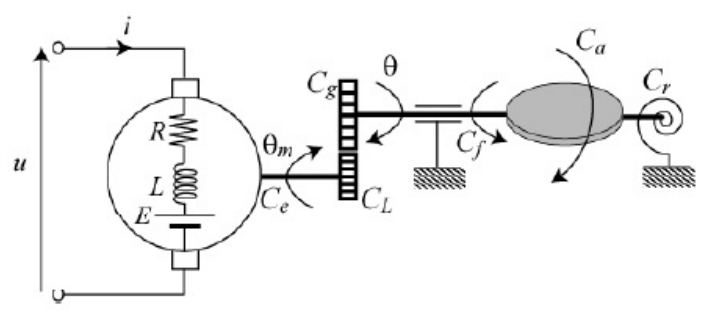

Figure 3: Electronic throttle system

The electrical part of this system is modeled by 41, $[20,21]$.

$$
u(t)=R i(t)+L \frac{d}{d t} i(t)+k_{v} \omega_{m}(t)
$$

$L$ is the inductance, $R$ the resistance, $u(t)$ and $i(t)$ the voltage and the armature current respectively, $k_{v}$ a constant electromotive force and $\omega_{m}(t)$ the motor rotational speed.

The mechanical part of the throttle is modeled by a gear reducer, characterized by its reduction ratio $\gamma$ such as 42

$$
\gamma=\frac{C_{g}}{C_{L}}
$$

$C_{L}$ is the load torque and $C_{g}$ the gear torque.

The mechanical part is modeled according to $(43)$ and (44), such that, [20,21]

$$
\begin{aligned}
J \frac{d}{d t} \omega_{m}(t) & =C_{e}-C_{f}-C_{r}-C_{a} \\
\frac{d}{d t} \theta(t) & =(180 / \pi / \gamma) \omega_{m}(t)
\end{aligned}
$$

$\theta(t)$ is the throttle plate angle, $C_{e}$ the electrical torque, $C_{f}$ the torque caused by mechanical friction, $C_{r}$ the spring torque, $C_{a}$ the torque generated by the airflow and $J$ the overall moment of inertia.

The electrical torque is defined by

$$
C_{e}=k_{e} i(t)
$$

where $k_{e}$ is a constant.

The electronic throttle valve involves two complex nonlinearities due to $C_{r}$ and $C_{f}$, given by their static characteristics, [15]

- a dead zone in which the control voltage signal has no effect on the nominal position of the valve plate,

- two hysteresis combined with a saturation, due to the valve plate movement, limited by the maximum and the minimum angles.

The static characteristic of the nonlinear spring torque $C_{r}$ is defined by

$$
C_{r}=\frac{k_{r}}{\gamma}\left(\theta-\theta_{0}\right)+D \operatorname{sgn}\left(\theta-\theta_{0}\right)
$$

for $\theta_{\min } \leqslant \theta \leqslant \theta_{\max }, k_{r}$ is the spring constant, $D$ a constant, $\theta_{0}$ the default position and $\operatorname{sgn}($.$) the following$ signum function

$$
\operatorname{sgn}\left(\theta-\theta_{0}\right)=\left\{\begin{array}{c}
1, \text { if } \theta \geqslant \theta_{0} \\
-1, \text { else }
\end{array}\right.
$$

The friction torque function $C_{f}$ of the angular velocity of the throttle plate can be expressed as

$$
C_{f}=f_{v} \omega+f_{c} \operatorname{sgn}(\omega)
$$

where $f_{v}$ and $f_{c}$ are two constants.

By substituting in equation (43), the expressions $C_{e}$, $C_{f}$ and $C_{r}$ and by neglecting the torque generated by the airflow $C_{a}$, the two nonlinearities $\operatorname{sgn}\left(\theta-\theta_{0}\right)$ and $\operatorname{sgn}(\omega)$ and the two constants $\frac{k_{r}}{\gamma} \theta_{0}$ and $f_{v}$, the studied system is linear then it can be modelized by the following transfer function $H(s)$ 49, [21]

$$
H(s)=\frac{(180 / \pi / \gamma) k_{e}}{J L s^{3}+J R s^{2}+\left(k_{e} k_{v}+L k_{s}\right) s+R k_{s}}
$$

with $k_{s}=\left(180 / \pi / \gamma^{2}\right) k_{r}$ and $s$ the Laplace operator; the identified parameters of this system are given in Table 2 at $25^{\circ} \mathrm{C},[20]$.

\begin{tabular}{|c|c|c|}
\hline Parameters & Values & Units \\
\hline$R$ & 2.8 & $\Omega$ \\
\hline$L$ & 0.0011 & $\mathrm{H}$ \\
\hline$k_{e}$ & 0.0183 & $\mathrm{N.m} / \mathrm{A}$ \\
\hline$k_{v}$ & 0.0183 & $\mathrm{v} / \mathrm{rad} / \mathrm{s}$ \\
\hline$J$ & $4 \times 10^{-6}$ & $\mathrm{~kg} . \mathrm{m}^{2}$ \\
\hline$\gamma$ & 16.95 & - \\
\hline
\end{tabular}

Table 2: Model's parameters

Recent work has shown that the ETV can be modeled by two linear models identified from the default position of the throttle plate for two values of the parameter $k_{s}$ and for the sampling period $T_{e}=0.002 s$, [21] 
- a model representing the position of the plate above the position by default for: $k_{s}=1.877 \times$ $10^{-4} \mathrm{~kg} \cdot \mathrm{m}^{2}$, then the corresponding discrete-time transfer function $H_{1}\left(q^{-1}\right)$ given by 50 ,

$H_{1}\left(q^{-1}\right)=\frac{0.007480 q^{-1}+0.01334 q^{-2}+0.0007376 q^{-3}}{1-1.948 q^{-1}+0.954 q^{-2}-0.006152 q^{-3}}$

- a model representing the position of the plate below the position by default for: $k_{s}=1.384 \times$ $10^{-3} \mathrm{~kg} \cdot \mathrm{m}^{2}$, then the corresponding discrete-time transfer function $\mathrm{H}_{2}\left(q^{-1}\right)$ given by 51 ,

$$
H_{2}\left(q^{-1}\right)=\frac{0.007479 q^{-1}+0.01333 q^{-2}+0.0007376 q^{-3}}{1-1.946 q^{-1}+0.954 q^{-2}-0.006152 q^{-3}}
$$

\subsection{Simulation results}

In order to test the proposed fault tolerant control approach, tow models of an ETV in actuator fault and an unknown disturbance case are given as state space formulation as

$$
\begin{gathered}
x(k+1)=A_{j} x(k)+B_{j} u(k)+E_{j} d(k)+B_{j} f_{a}(k) \\
y(k)=C_{j} x(k)
\end{gathered}
$$

where the parameter matrices and vectors of each model are given as

$$
\begin{gathered}
A_{1}=\left(\begin{array}{ccc}
1.948 & -0.954 & 0.006152 \\
1 & 0 & 0 \\
0 & 1 & 0
\end{array}\right) \\
A_{2}=\left(\begin{array}{ccc}
1.946 & -0.954 & 0.006152 \\
1 & 0 & 0 \\
0 & 1 & 0
\end{array}\right) \\
B_{1}=B_{2}=\left(\begin{array}{lll}
1 & 0 & 0
\end{array}\right)^{T} \\
C_{2}=\left(\begin{array}{lll}
0.007480 & 0.01334 & 0.0007376
\end{array}\right) \\
E_{1}=E_{2}=\left(\begin{array}{lll}
1 & 1 & 1
\end{array}\right)^{T}
\end{gathered}
$$

The studied system fulfills the conditions of Lemma 1.

$$
\left\{\begin{array}{l}
\operatorname{rank}\left(C_{1} E_{1}\right)=\operatorname{rank}\left(E_{1}\right)=1 \\
\operatorname{rank}\left(C_{2} E_{2}\right)=\operatorname{rank}\left(E_{2}\right)=1
\end{array}\right.
$$

then $\left(C_{1} A_{11}\right)$ and $\left(C_{2} A_{21}\right)$ are both observable.

By applying Theorem, the system is stable asymptotically for any switching signal for symmetric matrice $P$ definite positive given by 60 .

$$
P=\mathrm{e}-010\left(\begin{array}{ccc}
0.1429 & -0.0857 & 0.1903 \\
-0.0857 & 0.5085 & 0.0494 \\
0.1903 & 0.0494 & 0.4251
\end{array}\right)
$$

Then, the rest parameters of UIOs are calculated as below

$$
F_{1}=\left(\begin{array}{ccc}
-0.0266 & 0.8566 & -1.5415 \\
-0.0078 & 0.1954 & -0.3515 \\
0.0165 & -0.4329 & 0.7788
\end{array}\right)
$$

$$
\begin{gathered}
F_{2}=\left(\begin{array}{ccc}
-0.0233 & 0.8717 & -1.5349 \\
-0.0071 & 0.1998 & -0.3515 \\
0.0148 & -0.4384 & 0.7715
\end{array}\right) \\
T_{1}=\left(\begin{array}{lll}
0.9657 & -0.6187 & -0.3470 \\
-0.0343 & 0.3813 & -0.3470 \\
-0.0343 & -0.6187 & 0.6530
\end{array}\right) \\
T_{2}=\left(\begin{array}{lll}
0.9672 & -0.6196 & -0.3476 \\
-0.0328 & 0.3804 & -0.3476 \\
-0.0328 & -0.6196 & 0.6524
\end{array}\right) \\
K_{1}=\left(\begin{array}{l}
0.0002 \\
0.0031 \\
-0.0056
\end{array}\right), K_{2}=\left(\begin{array}{c}
0.0182 \\
0.0208 \\
-0.0389
\end{array}\right) \\
H_{1}=\left(\begin{array}{l}
46.3945 \\
46.3945 \\
46.3945
\end{array}\right), H_{2}=\left(\begin{array}{l}
46.4707 \\
46.4707 \\
46.4707
\end{array}\right)
\end{gathered}
$$

In this study, the method of tuning PID-type FLC parameters using GA is based on minimizing the ISE. If $y_{j}^{d}(k)$ is the desired flat trajectory and $\hat{x}_{j}(k)$ the estimated state, then we have

$$
\varepsilon_{j}(k)=-K_{c, j} \hat{x}_{j}(k)+y_{j}^{d}(k)
$$

For the ISE defined by

$$
I S E=\int_{0}^{t} \varepsilon_{j}^{2}(k) d t
$$

In this paper, the considered fitness function is taken as inverse of this error, i.e. the following performance index

$$
\text { fitness value }=\frac{1}{I S E}
$$

Then, the obtained optimum values of the input/output scaling factors $\left(K_{e}, K_{d}, \alpha, \beta\right)$, using genetic algorithm are given as follows: $K_{e}=0.2006, K_{d}=$ $0.8071, \alpha=0.1146$ and $\beta=0.2063$.

The desired discrete time flat trajectory $z_{j}^{d}(k)$, with $j=\{1,2\}$ can be computed according to the following polynomial form

$$
z_{j}^{d}(k)=\left\{\begin{array}{c}
\frac{c s t 1}{B_{j}(1)}, \text { if } 0 \leqslant k \leqslant k_{0} \\
\operatorname{Poly}_{1, j}(k), \text { if } k_{0}<k \leqslant k_{1} \\
\frac{c s t 2}{B_{j}(1)}, \text { if } k_{1}<k \leqslant k_{2} \\
\operatorname{Poly}_{2, j}(k), \text { if } k_{2}<k \leqslant k_{3} \\
\frac{c s t 1}{B_{j}(1)}, \text { if } k>k_{3}
\end{array}\right.
$$

where $\operatorname{cst} 1$ and $\operatorname{cst} 2$ are constant parameters, $k_{0}=3 s$, $k_{1}=6 s, k_{2}=10 s$ and $k_{3}=15 s$ are the instants of transitions, $B_{j}(1)$ is the static gain between the flat output $z_{j}(k)$ and the output signal $y_{j}(k)$ for each model and $\operatorname{Poly}_{1, j}(k)$ and $\operatorname{Poly}_{2, j}(k)$ are polynomials calculated using the technique of polynomial interpolation.

The desired trajectories $y_{1}^{d}(k)$ and $y_{2}^{d}(k)$ are then given in Figure 4 


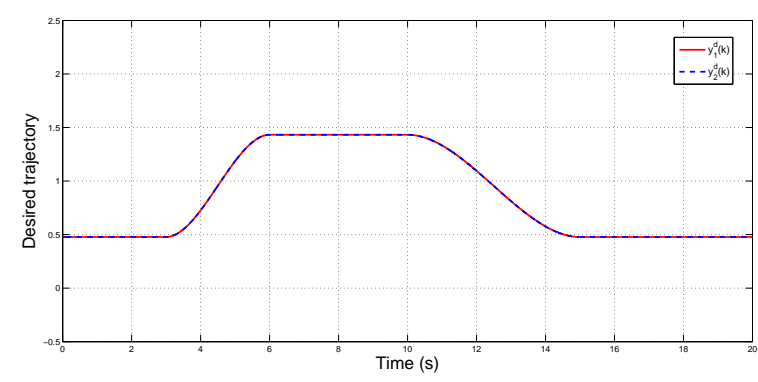

Figure 4: Desired trajectories $y_{1}^{d}(k)$ and $y_{2}^{d}(k)$

Let us consider the fault vector

$$
f_{a}(k)=\left[\begin{array}{l}
f_{a 1}^{T}(k) \\
f_{a 2}^{T}(k)
\end{array}\right]
$$

such as $f_{a 2}=0$ and $f_{a 1}$ defined as follows

$$
f_{a 1}(k)=\left\{\begin{array}{cc}
0, & k \in[0,10] \\
-0.1 \sin (0.314 k), & k \in] 10,13] \\
0, & k \in] 13,20]
\end{array}\right.
$$

The simulation results illustrated in Figure 5 and Figure 6. show some oscillations for the outputs signals and for the tracking error due to the unknown input. We remark that the system's responses with and without FTC track desired trajectories with disturbances rejection.

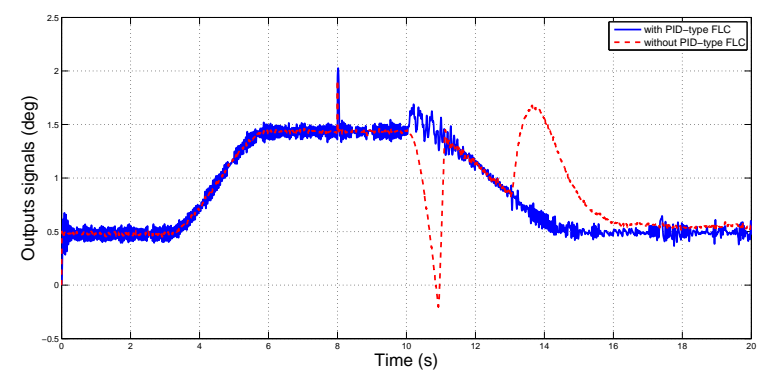

Figure 5: System outputs in actuator fault case with and without PID-type FLC

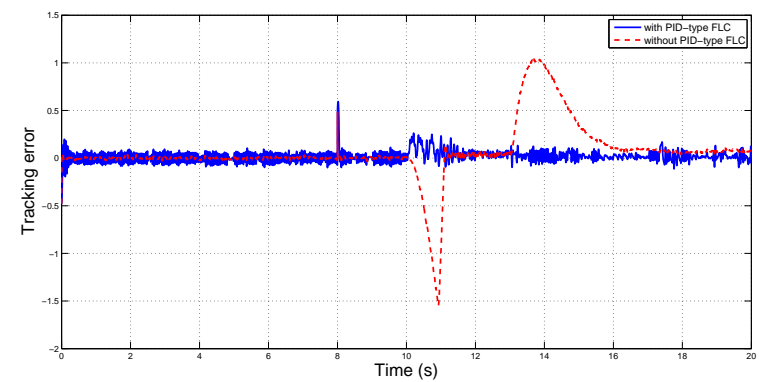

Figure 6: Tracking error in actuator fault case with and without PID-type FLC

At the time of actutor faults occurrence $k=10 \mathrm{~s}$, the system's behavior was changed. The system without
FTC becomes unstable, whereas for the same reference input and by using the FTC, the system remains stable and the tracking error have a small deviation from zero which shows the effectiveness of the proposed FTC approach.

Figure 7, Figure 8 and Figure 9 show the residual values generated using UIOs and the switched signal. The switching between the two models is acheived based on residual values comparision.

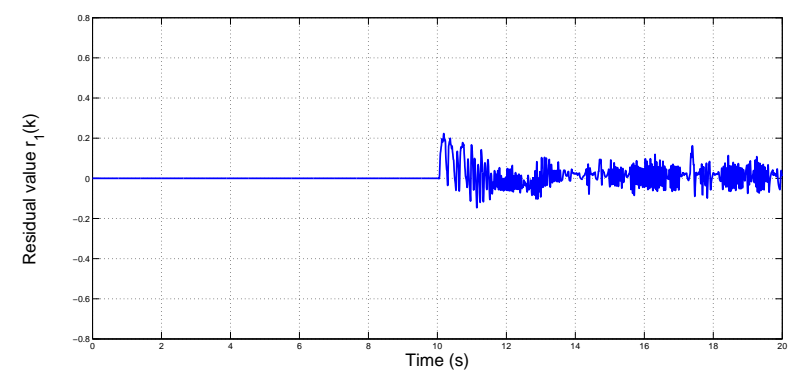

Figure 7: Residual value $r_{1}(k)$ in actuator fault case with PIDtype FLC

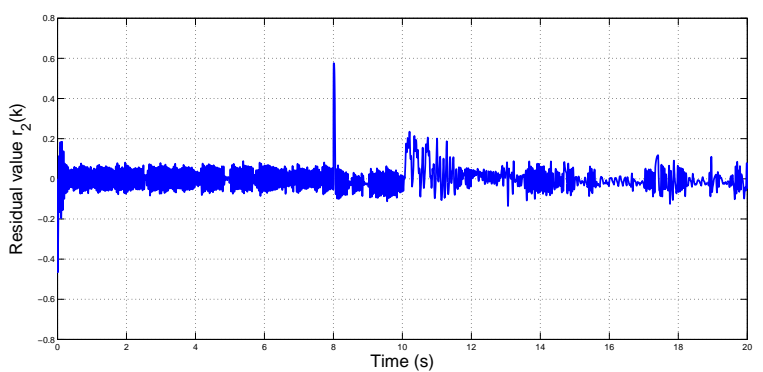

Figure 8: Residual value $r_{2}(k)$ in actuator fault case with PIDtype FLC

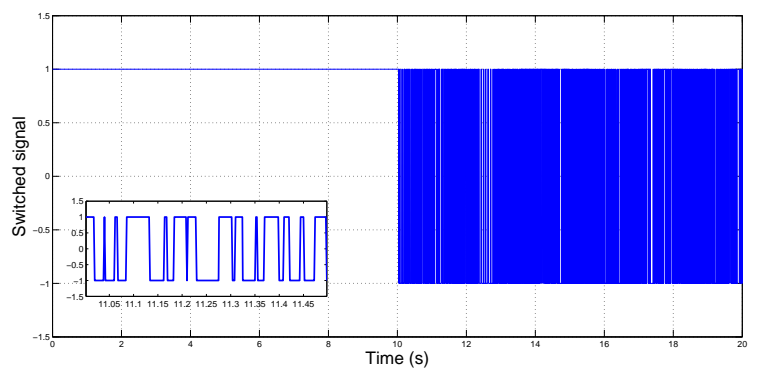

Figure 9: Switched signal in actuator fault case with PID-type FLC

\section{Conclusion}

In this paper, a new fault tolerant control law based on PID-type FLC is designed for the nonlinear complex system ETV, modelized by a multimodel structure. The approach is based on the use of a reference 
model generated using the flatness property. The proposed control law is, then, designed to minimize the error between the desired flat trajectory and the estimated state, generated using UIOs, even in the presence of actuator faults based on minimizing the ISE.

\section{References}

1. B.Y. Huo, Y.M. Li and S.C. Tong, Fuzzy adaptive faulttolerant output feedback control of multi-input and multioutput non-linear systems in strict-feedback form, IET Control Theory and Applications, 6, 2704-2715, 2012.

2. Q.K. Shen, B. Jiang and V. Cocquempot, Fuzzy logic system-based adaptive fault-tolerant control for nearspace vehicle attitude dynamics with actuator faults, IEEE Transactions on Fuzzy Systems, 21, 289-300, 2013.

3. S.C. Tong, B.Y. Huo and Y.M. Li, Observer-based adaptive decentralized fuzzy fault-tolerant control of nonlinear large-scale systems with actuator failures, IEEE Transactions on Fuzzy Systems, 2,1-15, 2014.

4. D. Ichalal, B. Marx, J. Ragot and D. Maquin, Fault tolerant control for Takagi-Sugeno systems with unmeasurable premise variables by trajectory tracking, IEEE International Symposium on Industrial Electronics (ISIE), Bari, 2010 .

5. M. Blanke, M. Kinnaert, J. Lunze and M. Staroswiecki, Diagnosis and Fault-Tolerant Control, Springer-Verlag Berlin Heidelberg, 2006.

6. R. Nouailletas, D. Koeing and E. Mendes, LMI design of a switched observer with model uncertainty: Application to a hysteresis system, Proc. of the 46th IEEE CDC, New Orleans, 6298-6303, 2007.

7. D. Wang, W. Wang and P. Shi, Robust fault detection for switched systems with state delays, IEEE Trans. on Systems and Cybernetics-B, 39, 800-805, 2009.

8. H. Yingxue, T. Shaocheng and L. Yongming, Adaptive fuzzy backstepping control for a class of switched nonlinear systems with actuator faults, International Journal of Systems Science, 47, 3581-3590, 2015.

9. A. Benzaouia, M. Ouladsine, A. Naamane and B. Ananou, Fault detection for uncertain delayed switching discretetime systems, International Journal of Innovative Computing, Information and Control, 8, 1-11, 2012.

10. M. Oudghiri, M. Chadli and A. El Hajjaji, Robust observer-based fault tolerant control for vehicle lateral dynamics, International Journal of Vehicle Design, 48, 173$189,2008$.
11. D. Ichalal, B. Marx, D. Maquin and J. Ragot, Nonlinear observer based sensor fault tolerant control for nonlinear systems, 8th IFAC Symposium on Fault Detection, Supervision and Safety of Technical Processes, Mexico, 2012.

12. M. Benrejeb, Stability Study of Two Level Hierarchical Nonlinear Systems, 12th IFAC Symposium on Large Scale Systems: Theory and Applications, 43 (8), 30-41 Villeneuve d'Ascq, 2010.

13. J. Chen, R.J. Patton and H.Y. Zhang, Design of unknown input observers and robust fault detection filters, International Journal of Control, 63, 85-105, 1996.

14. W. Jiawei, S. Yi and Z. Miao, Fault Detection for Linear Switched Systems Using Unknown Input Observers, Proceedings of the 33rd Chinese Control Conference, Nanjing, 2014.

15. W. Gritli, H. Gharsallaoui and M. Benrejeb, Fault Detection Based on Unknown Input Observers for Switched Discrete-Time Systems, Int. Conf. on Advanced Systems and Electrical Technologies IC-ASET, Hammamet, 2017.

16. S. Bouallègue, M. Ayadi, J. Haggége and M. Benrejeb, A PID type fuzzy controller design with flatness-based planning trajectory for a DC drive, IEEE International Symposium on Industrial Electronics, Cambridge, 1197-1202, 2008.

17. M. Guzelkaya, I. Eksin and E. Yesil, Self-tuning of PIDtype fuzzy logic controller coefficients via relative rate observer, Engineering Applications of Artificial Intelligence, 16, 227-236, 2003.

18. W. Gritli, H. Gharsallaoui and M. Benrejeb, PID-type Fuzzy Scaling Factors Tuning Using Genetic Algorithm and Simulink Design Optimization for Electronic Throttle Valve, International Conference on Control, Decision and Information Technologies CoDIT, Malta, 2016.

19. M. Fliess, J. Lévine, P. Martin and P. Rouchon, On differentially flat non linear systems, In Proc. IFACSymposium NOLCOS, pp. 408-413, Bordeaux, 1992.

20. M. Lebbal, H. Chafouk, G. Hoblos and D. Lefebvre, Modelling and Identification of Non-Linear Systems by a Multimodel Approach: Application to a Throttle Valve, International Journal Information and Systems Science, 3, 67-87, 2007.

21. C. Yang, Model-based analysis and tuning of electronic throttle controllers, Visteon Corporation, SAE 2004 World Congress \& Exhibition, 63-67, 2004. 